Pbilosophia Philosophia Scientiæ

Scientia

Travaux d'histoire et de philosophie des sciences

9-2 | 2005

Aperçus philosophiques en logique et en mathématiques

\title{
Independence and games
}

Denis Bonnay

\section{(2) OpenEdition}

Journals

Electronic version

URL: http://journals.openedition.org/philosophiascientiae/547

DOI: $10.4000 /$ philosophiascientiae.547

ISSN: $1775-4283$

Publisher

Éditions Kimé

Printed version

Date of publication: 1 November 2005

Number of pages: 295-304

ISBN: 2-84174-379-9

ISSN: 1281-2463

Electronic reference

Denis Bonnay, "Independence and games", Philosophia Scientiæ [Online], 9-2 | 2005, Online since 15

June 2011, connection on 16 January 2021. URL: http://journals.openedition.org/

philosophiascientiae/547 ; DOI: https://doi.org/10.4000/philosophiascientiae.547 


\author{
Denis Bonnay \\ IHPST / University of Paris I (France).
}

Résumé : La logique IF prétend constituer une alternative à la logique classique du premier ordre : en libéralisant les schémas de dépendance entre quantificateurs, elle mènerait à leur terme les idées sous-jacentes à la logique classique. Mais les jeux de Hintikka ne constituent pas la seule manière possible de fournir une sémantique pour l'indépendance : on pourrait au contraire vouloir le faire dans le cadre d'une sémantique récursive avec des quantificateurs de Henkin. Nous présentons ici quelques arguments techniques et philosophiques en faveur de IF, en montrant pourquoi son concept d'indépendance, élargi aux connecteurs, peut prétendre être pleinement général, et en montrant en quel sens la logique IF traite l'indépendance de manière analytique. Ce dernier point est réalisé à travers une explicitation du contenu épistémique de IF, sous la forme d'une traduction partielle dans la logique modale.

Abstract: Hintikka and Sandu have developed IF logic as a genuine alternative to classical first-order logic: liberalizing dependence schemas between quantifiers, IF would carry out all the ideas already underlying classical logic. But they are alternatives to Hintikka's game-theoretic approach; one could use instead Henkin quantifiers. We will present here some arguments of both technical and philosophical nature in favor of IF. We will show that its notion of independence, once extended to connectives, can indeed claim to be fully general, and that IF logic provides an analysis of independence patterns. This last point will be argued for thanks to an explanation of the epistemic content of IF, through a partial translation into modal logic.

Philosophia Scientice, 9 Cahier 2, 2005, 291-300. 


\section{Introduction}

IF logic is a logic which extends first-order logic by removing restrictions on the possible dependence patterns between quantifiers. The claim developed in [Hintikka 1996] according to which IF logic is the classical logic is based on the idea that no more is required to understand IF logic than to understand FO logic. Once one has adopted a game semantics, the liberalization of dependence patterns is very natural : it corresponds to the shift from perfect to imperfect information games. But one could as well use Henkin quantifiers to give a recursive semantics for independence. Putting aside the partiality phenomena, which could also be mimicked, the question cannot be settled on the level of pure expressivity, and arguments are likely to boil down to the fact that IF approach is more natural, elegant, or intuitive. We are going to try to give some precise content to this feeling. Namely, we will discuss two points : the claim of generality and the idea that IF gives a true analysis of independence. We will make thus some technical points about the use of slash and links with modal logic.

\section{Defining information partitions}

To begin with, we would like to examine the idea that IF logic is fully general. Can the concept of independence apply freely to all connectives and why could we say that, from this point of view, IF logic is as general as it could be? Let's go back to the definition of semantic games.

In general, an extensive game is a 6 -uplet $\left\langle N, H, Z, J, P,(I)_{i \in N}\right\rangle$ :

$-N$ is the set of players

- $H$ is the set of histories, i.e. a set of sequences containing the empty sequence and which is closed by prefix

$-Z \subseteq H$ is the set of terminal histories

- $\mathrm{J}$ is a function from $H / Z$ to $N$ which determines the players' turn to play

- $\mathrm{P}$ is the payoff function

- $(I)_{i}$ is a partition of $\{h \in H / J(h)=i\}$

A formula $\phi$ in normal form for negation and a model $M$ define a labeled game $\mathrm{G}_{\phi} . \mathrm{N}$ is $\{\forall, \exists\}$. $\mathrm{H}$ is defined recursively together with $\mathrm{J}$ and a labeling function 1 :

$-<>\in H, l(<>)=\phi$

- if $h \in H$ and $\mathrm{l}(\mathrm{h})=\psi \wedge \theta$, then $J(h)=\forall, h \frown L \in H, l(h \frown L)=$ $\psi, h \frown R \in H$, and $l(h \frown R)=\theta$ 
- if $h \in H$ and $\mathrm{l}(\mathrm{h})=\exists x \psi$, then $J(h)=\exists$, and for all $a \in|M|, h \frown$ $a \in H, l(h \frown a)=\psi[x:=a]$.

$\mathrm{Z}$ is the subset of $\mathrm{H}$ whose histories are labeled by an atomic formula.

$\mathrm{P}$ gives a terminal history $\mathrm{h}$ the value 1 or 0 , according to the truth or falsity of the atomic formula labelling $\mathrm{h}$ in the model.

If $\phi$ is a formula of classical logic, the information sets of the players are trivially defined as the partition of $J^{-1}(\exists / \forall)$ consisting only of singletons. But the situation is not always so simple :

Problem 1. How does one define $I_{\exists}$ and $I_{\forall}$ when $\phi$ belongs to IF logic?

The idea is that the players' information partitions will be less finegrained; two histories $h$ and $h$ ' belonging to the same information set are so to say mixed up by the player so that for any strategy $\sigma$ one must have $\sigma(h)=\sigma\left(h^{\prime}\right)$. If one allows independence only with respect to quantifiers, everything works well, but it is not so clear that one can define independence in full generality, i.e. with respect to propositional connectives as well. Two problems were raised by Sandu and van Benthem [van Benthem 2001] for the first one, and by Hodges [Hodges 1997] for the second one :

1) meaningless syntactical possibilities, like in

$P a \vee(\phi \wedge / \vee \psi)$

Here the slash should tell that the second move by $\forall$ bélard is independent of ヨloïse's choice for the disjunction. But what this should mean is not very clear because $\forall$ bélard must know she has chosen the second disjunct as far as she knows she has to play.

2) counter-intuitive meaning of some sentences when independence is defined with respect to the skolemization procedure, like in $\exists x_{/ \wedge} \phi(x) \wedge \exists x_{/ \wedge} \psi(x)$

Here one would expect something equivalent to $\exists x(\phi(x) \wedge \psi(x))$ : $\exists$ loïse chooses an individual without knowing $\forall$ bélard's choice for the conjunction. But the skolemization leads to $\exists x_{1} \exists x_{2}\left(\phi_{1}(x) \wedge \psi_{2}(x)\right)$,

where no meaningful independence can be plugged in.

Sandu (Independence-friendly languages, unpublished manuscript) has suggested to overcome the problem by construing connectives as quantifiers over formulas in Vaugth's manner. We shall see this is unnecessary, because in order to solve the problem, it is sufficient to give a good definition of the information partitions. 
Let head/tail be a function giving the head/tail of a sequence, $m c$ a function giving the main connective of a formula and slash be a partial function giving us for a history $\mathrm{h}$ the connectives of $\phi$ for which the main connective of $\mathrm{l}(\mathrm{h})$ is slashed.

Definition 2. Given two histories $h$ and $h^{\prime}$ in $H$ such that $J(h)=$ $J(h \prime)=i$ and length $(h)=$ length $\left(h^{\prime}\right), h R_{i} h^{\prime}$ iff

for all initial segments $s$ and s' of same length of $h$ and $h$ ',

1. the types of $m c(l(s))$ and $m c(l(s /))$ are the same (slashes included)

2. either head $(s)=$ head $\left(s^{\prime}\right)$ or $m c(l(\operatorname{tail}(s))) \in \operatorname{slash}(h)$ and $m c\left(l\left(\operatorname{tail}\left(s^{\prime}\right)\right)\right) \in \operatorname{slash}\left(h^{\prime}\right)$

Condition 1. says that the histories should correspond to actions of the same type and condition 2. says that if two actions are different, then they should correspond to slashed connectives.

One can easily check that the $R_{i}$ are equivalence relations leading to information partitions which give a satisfactory interpretation to the problematic occurrences of slashes. In the first case, we simply have no independence at all, because condition 1. is not satisfied, and in the second case, both conditions will be satisfied at the critical nodes, so that the truth-conditions of the formula are the intended ones. More generally, one could check that the information partitions are well defined according to the usual game-theoretic criteria. So we have well-defined games for independence with respect to connectives, without being compelled to use tricks such as interpreting disjunction or conjunction as quantification over formulas.

\section{Generality}

The main advantage of our previous definition is to pave the way for an answer to the next question.

Problem 3. Could we have new information-defining operators like the slash?

The shift from classical logic to IF logic is obtained by adding a new operator, the slash. Its semantic interpretation is totally different from the usual connectives. Whereas they can be seen as game-forming operators, the action of the slash does not modify the tree-structure of the game but bears on the information-structure. As a consequence, one could ask if the claim of Hintikka (bringing the ideas behing FO 
quantification to their full generality by freeing oneself from arbitrary syntactic limitations) is justified, that is if IF logic is fully general with respect to arbitrary possible information-defining operators.

Let's go back to condition 2., it says that a certain relation must hold between subhistories, and which relation must hold depends on slash(h) and $\operatorname{slash}\left(\mathrm{h}^{\prime}\right)$, that is on the information patterns allowed. We could redefine a function $\operatorname{slash}(h, s)$ giving the information-defining operator qualifying the main connective of $l(h)$ with respect to the connective prompting the action leading to the position $s$, and then condition 2 . would read :

\section{head $(s) R_{I N D}$ head $(s)$}

with $\left(I N D=\operatorname{slash}(h\right.$, head $\left.(s))=\operatorname{slash}\left(h^{\prime}, \operatorname{head}(s)\right)\right)$. Information-defining operators are semantically interpreted as binary relations on models (to be precise, as functions assigning to each model such a binary relation). This is in the spirit of what happens in generalized quantifier theory where for example unary quantifiers are seen as subsets of the powerset of the models. Thus $R_{/}(M)$ is the universal relation on $|M|$ whereas $R_{\text {blank }}(M)$ (corresponding to the absence of slash) is the identity on $|M|$. For example, the formula $\forall x \exists y \exists z / x \phi(x, y, z)$ can be construed as $\forall x \exists y_{\text {blank }(x)} \exists z_{/ x, \operatorname{blank}(y)} \phi(x, y, z)$.

So our question can be restated like this : what kind of binary relations on models can behave like information defining operators for a game-theoretic logic. The following conditions seem reasonable conditions for any $R_{I N D}$ :

Game-theoretic condition $R_{I N D}(M)$ must be an equivalence relation on $M$. If this is not the case, the information sets induced by $R_{I N D}(M)$ will not form a partition of $J^{-1}(i)$, but then it is no more possible to say what a uniform strategy must be.

Logical condition As far as logic is expected to be topic-neutral, $R_{I N D}(M)$ must be closed under permutation on $|M|$ (see Sher 1991 for a philosophical motivation in the context of generalized quantifier theory) If this is not the case, $R_{I N D}(M)$ will make some distinctions among objects in M, something a logical operator should not do.

Semantic condition $\mathrm{R}_{I N D}$ must be homogenous, in the sense that it should mean the same across models (exactly for the same reasons for which we do not want a quantifier which would mean $\exists$ on models of a given size and $\forall$ on models of other sizes).

Now, it is well known that there are only four binary relations satisfying the logical condition, the universal relation, the empty relation, identity 
and diversity. Only two out of these four, the universal relation and identity, are equivalence relations. Then, by the semantic condition, we require that $\mathrm{R}_{I N D}$ will be interpreted either by the universal relation on all models, or by the identity on all models. So the answer to our second question is yes :

Proposition 4. IF logic is as general as possible with respect to independence patterns

This result obviously depends on the kind of generalizations we have considered : we have established only that IF logic is fully general with respect to independence operators, where independence operators are binary relations on domains ruling the independence patterns essentially between quantifier choices. One could devise other kind of extensions, for example by adding a third player as [Pietarinen 2002] has proposed. Of course this should be no surprise. But in these sort of cases, it would be more difficult to defend the idea that the extension generalizes on ideas already underlying classical FO-logic. On the contrary, one could argue that this easy result shows indeed that full generality has been reached relatively to the idea of arbitrary dependence patterns.

\section{Analysis}

Hintikka and Sandu [Hintikka \& Sandu 1994] repeatedly argue against the treatment of independence by means of Henkin quantifiers by pointing out that Henkin quantifiers do not treat independence separately but isolate blocks of quantifiers, whereas the game semantics provides a step by step account of independence. In which respect does IF provide a true analysis of the independence phenomena? We have seen that this is done by keeping a step by step construction of the games associated to formulas while adding an information structure on the game. This means that indepence is handled by restricting the knowledge of the players.

Problem 5. Can one give a formal account of the epistemic content of IF logic?

One could imagine two kinds of reductions of IF sentences to epistemic sentences.

- a full reduction Given an IF sentence $\phi$ and a model M, we give an epistemic sentence $\phi^{\prime}$, build from $\mathrm{F}$ and an epistemic model M', build from $M$ such that $M \vDash \phi$ iff $M^{\prime} \vDash \phi^{\prime}$. 
- a partial reduction One only tries to isolate the epistemic content of IF sentences.

I think that the second approach is both more manageable and more meaningful, sticking to the idea that the shift from FO logic to IF logic corresponds to the addition of epistemic constraints.

Given an IF formula $\phi$ and a model $A$, we seek :

- a classical formula $\phi^{*}, \phi$ classical counterpart.

- a Kripke structure $M$, describing the game $G_{\phi, A}$.

- an epistemic sentence $\psi_{i}$ describing the knowledge needed to win for the player i.

such that $\phi^{*}, M$ and $\psi_{i}$, built from $\phi$ and $A$ satisfy :

$A \vDash^{i} \phi$ iff $\left[A \vDash^{i} \phi^{*}\right.$ and $\left.M \vDash \psi_{i}\right]$

where $\models^{i}$ means the existence of a winning for $i$.

The proof is rather simple, let's give the ideas first. $\phi^{*}$ is simply obtained from $\phi$ by dropping the slashes. In the spirit of [van Benthem 200 ?], ${ }^{1}$ the Kripke structure $M$ is simply the game tree $G_{\phi, M}$, the accessibilty relations corresponding to the equivalence relations induced by the information partitions, the atoms of the propositionnal modal language are just names for the nodes of the tree, valuated the intuitive way.

A bit more technical is the design of $\psi_{i}$. The idea is that $\psi_{i}$ expresses the knowledge needed to win, when $M$ expresses the knowledge actually possessed by the players. As a consequence, $\psi_{i}$ will be valid on $M$ if the knowdelge granted to the player $\mathrm{i}$ in the imperfect game corresponding to $\phi$ is enough for $\mathrm{i}$ to have a winning strategy on $G_{\phi, M} \cdot \psi_{i}$ is obtained through variation of the information partitions granted to i in $G_{\phi, M}$, suitably filtered to simplify $\psi_{i}$. We shall note that $\psi_{i}$ will require use of infinite conjunctions and disjunctions when $G_{\phi, M}$ is itself infinite. To get things simpler, we will suppose that $G_{\phi, M}$ is finite (the extension to the infinite case is straightforward through the shift to infinitary propositionnal modal logic).

To begin with, we explain how to construct $\psi_{i}$. Let $P$ be a partition of a set, we denote by $R_{P}$ the equivalence relation induced by that partition. We define the set of the alternative information structure for the player $i$ :

$I P_{i}=\left\{P / P\right.$ is a partition of $J^{-1}(\{i\})$ and for all h, h' $\in H, h R_{p} h^{\prime}$ implies length $\left.(\mathrm{h})=\operatorname{length}\left(\mathrm{h}^{\prime}\right)\right\}$

1. [van Benthem 2002] and [van Benthem 200 ?] offer a much wider application of modal logic to the analysis of extensive games; the result we present in this section stems out of a much more modest project, namely to make clear the epistemic component of IF logic. 
This gives a family $G_{i}$ of games which differ of G only on $I_{i}$ :

$$
G_{i}=\left\{G^{\prime} / \text { there is a } P \in I P_{i} \text { such that } G^{\prime}=G\left[P / I_{i}\right]\right\}
$$

Among all these alternative information structures, one can distinguish the winning ones :

$W I P_{i}=\left\{P \in I P_{i} /\right.$ there is a winning strategy for player $i$ on $\left.G_{p}=G\left[P / I_{i}\right]\right\}$

It is possible to work a bit on $W I P_{i}$ to reduce its size, although this is not essential for the proof. One could focus on minimal winning partitions, in the following sense of minimal. Let $P, P^{\prime} \in W I P_{i}, P \precsim P^{\prime}$ iff $_{\text {def }} \forall h, h^{\prime} \in H, h R_{P^{\prime}} h^{\prime} \rightarrow h R_{P} h^{\prime}$. Intuitively, $\precsim$ means 'less finegrained than'. It is very easy to check that $\precsim$ is an ordering on $W I P_{i}$. In the general case, $\precsim$ is not a total order. We can then restrict ourself to the minimal winning information partitions for $i$ :

\section{$M W I P_{i}=\left\{P \in W I P_{i} / P\right.$ is $\precsim-$ minimal on $\left.W I P_{i}\right\}$}

We can note that they are two distinguished partitions, $P^{*}$ in which all the histories of the same depth are mixed up, and $P^{* *}$ which consist only in singletons. If we see $\precsim$ as an order on $I_{i}, P^{*}$ and $P^{* *}$ are respectively the smallest and the greatest elements. But there might be no smallest element in $W I P_{i}$; this is the case if there is no winning strategy for player $i$ on $P^{*}$.

Let $P_{0} \ldots P_{n}$ be the partitions in $W I P_{i}$ and $h_{0} \ldots h_{z}$ the histories in $H$. Let $\mathrm{L}$ be the modal propositional language whose atoms are $h_{0} \ldots h_{z}$. Let $k$ be an integer no greater than $n$. If $0 \leq l, m \leq z$, we define

$$
F_{l, m} \equiv_{\text {def }}\left(K_{i} \sim h_{l}\right) \vee\left(K_{i} \sim h_{m}\right)
$$

and given $\Gamma_{k}=\left\{F_{l, m} / 0 \leq l, m \leq z\right.$ and $\left.\sim h_{l} R_{P_{k}} h_{m}\right\}$, we shall denote by $\theta_{k}$ the conjunction of the formulas in $\Gamma_{k}$. It could be possible to decrease the size of $\Gamma_{k}$ using tautologies of the propositionnal calculus.

The idea behind this construction is that $\theta_{k}$ is a description of $P_{k}$ in so far as it tells us between which situations the player $i$ can distinguish when its information structure is given by $P_{k}$. Intuitively, to know that $\sim h_{l}$ or to know that $\sim h_{m}$ is intended to mean being able to distinguish between the two positions $h_{l}$ and $h_{m}$.

We can now define $\psi_{i}$ :

$$
\psi_{i} \equiv \underset{0 \leq k \leq n}{\vee} \theta_{k} \text { if } W I P_{i} \text { is not empty and } \top \text { if } W I P_{i} \text { is empty. }
$$

As we said before, the definition of the Kripke structure $M$ is quite natural. The set $H$ of histories is the domain on which the accesibility relation $R_{i}$ is the equivalence relation induced by $I_{i}$. We have to evaluate the atoms $h_{0} \ldots h_{z}$, this is done by putting simply $h_{i} \in \operatorname{Val}\left(h_{j}\right)$ iff $i=j$. 
Let's check that

Proposition 6. $A \vDash^{i} \phi$ iff $\left[A \vDash^{i} \phi^{*}\right.$ and $\left.M \vDash \psi_{i}\right]$

We shall show first that $P_{k} \precsim I_{i}$ iff $M \vDash \theta_{k}$.

- $P_{k} \precsim I_{i}$ implies $M \vDash \theta_{k}$. Assume $P_{k} \precsim I_{i}$ and let $F_{l, m}$ be one of the conjuncts of $\theta_{k}$. By construction of $\theta_{k}$, this means that $\sim h_{l} R_{k} h_{m}$. As $P_{k} \precsim I_{i}$, we have also $\sim h_{l} R_{i} h_{m}$. It follows that $M \vDash F_{l, m}$ : let $h$ be any history. Suppose that $M, h \not \models F_{l, m}$ This means that both $h_{l}$ and $h_{m}$ are $R_{i}$-accessible from $h$, but as $R_{i}$ is an equivalence relation, this implies that $h_{l}$ and $h_{m}$ are themselves $R_{i}$-related, which gives us a contradiction.

- $M \vDash \theta_{k}$ implies $P_{k} \precsim I_{i}$. Assume $M \vDash \theta_{k}$. Let $h_{l}$ and $h_{m}$ be any histories such that $\sim h_{l} R_{k} h_{m}$, we show that we have $\sim h_{l} R_{i} h_{m}$ as well. By hypothesis, $M \vDash\left(K_{i} \sim h_{l}\right) \vee\left(K_{i} \sim h_{m}\right)$. Assume that $h_{l} R_{i} h_{m}$. We have then $M, h_{l} \not \models\left(K_{i} \sim h_{l}\right) \vee\left(K_{i} \sim h_{m}\right)$ because $M, h_{l} \vDash h_{l}$ and $M, h_{m} \vDash h_{m}$, which gives us a contradiction.

The desired result follows :

- $A \models^{i} \phi$ implies $\left[A \vDash^{i} \phi^{*}\right.$ and $\left.M \vDash \psi_{i}\right]$. It is quite obvious that $A \models^{i}$ $\phi$ implies $A \models^{i} \phi^{*}$. And if $A \models^{i} \phi$, we have that $I_{i} \in W I P_{i}$, so that there is a $P_{k} \in M W I P_{i}$ such that $P_{k} \precsim I_{i}$. We have then $M \vDash \theta_{k}$ and $M \vDash \psi_{i}$.

- $\left[A \models^{i} \phi^{*}\right.$ and $\left.M \vDash \psi_{i}\right]$ implies $A \models^{i} \phi$. As $A \models^{i} \phi^{*}, \psi_{i}$ is different from $\top$. This means that there is a $k$ such that $M \vDash \theta_{k}$. So there is a $P_{k} \in M W I P_{i}$ such that $P_{k} \precsim I_{i}$ for which the player $i$ has a winning strategy. It follows straightforwardly that $i$ has a winning strategy on $G$. Intuitively, if $i$ can win with a certain amount of information, he can win as well with less information, the winning strategy for $I_{i}$ being directly obtained from the one for $P_{k}$.

This result exactly gives what we were looking for : the existence of a winning strategy on the imperfect information game can be analysed in the existence of a winning strategy on the corresponding perfect information game plus additional knowledge. We may say that IF logic is implicitly epistemic, that our construction makes this epistemic content explicit, and that this gives a precise account of the way IF logic deals with independence. 


\section{References}

VAN BENTHEM, JOHAN

200 ? 'Hintikka Self-applied' : an essay on the epistemic logic of imperfect information games, to appear in Lewis Hahn, (ed.), Hintikka Volume, Library of Living Philosophers.

2002 Extensive Games as Process Models, Journal of Logic, Language and Information, vol 11 (3) : 289-313.

2001 Logic in Games, Lecture notes ILLC.

HiNTIKKA, JAAKKO

1996 The principles of mathematics revisited, Cambridge : Cambridge University Press.

HintikKa, JaAkKo, \& SAndu, Gabriel

1994 What is a quantifier?, Synthese, $98: 113-129$.

HODGES, WILFRID

1997 Compositional Semantics for a Language of Imperfect Information, Journal of the Interest Group in Pure and Applied Logics, 5 (4) : 539-563.

\section{Pietarinen Ahti}

2002 Independence-friendly logic and incomplete information, talk at PILM 2002, Nancy.

Sher, Gila

1991 The Bounds of Logic, Cambridge : MIT Press. 